\title{
Changing optical nonlinearities of homoleptic bis(phthalocyaninato) rare earth praseodymium double-decker complexes by the redox reaction
}

\author{
Boxiao Ren ${ }^{1}$, Ning Sheng ${ }^{2}$, Bing Gu ${ }^{1, *}$, Yong Wan ${ }^{1}$, Guanghao Rui ${ }^{1}$, Changgui \\ $\mathrm{Lv}^{1}$, and Yiping Cui ${ }^{1}$
}

${ }^{1}$ Advanced Photonics Center, Southeast University, Nanjing 210096, Jiangsu, China

${ }^{2}$ Key Laboratory of Inorganic Chemistry in Universities of Shandong, Department of

Chemistry and Chemical Engineering, Jining University, Qufu 273155,Shandong,

China

*Corresponding author: gubing@seu.edu.cn 
Abstract: We report the third-order nonlinear optical properties of the homoleptic bis(phthalocyaninato) rare earth double-decker complex $\operatorname{Pr}(\mathrm{OOPc})_{2}(\mathbf{1})$, the oxidized species $\left[\mathrm{Pr}(\mathrm{OOPc})_{2}\right]^{+}(2)$, and the reduced species $\left[\mathrm{Pr}(\mathrm{OOPc})_{2}\right]^{-}(3)$, by performing the femtosecond-pulsed Z-scan experiments at the wavelength of $800 \mathrm{~nm}$. It is found that the second-order hyperpolarizability in complex molecules decreases in the order of 1 $>$ 2 $>$ 3. More interestingly, the observed nonlinear absorption process changes from two-photon absorption in the oxidized 2 to saturable absorption in the reduced 3 . The results demonstrate that the nonlinear optical properties of sandwich-type phthalocyanine rare earth complexes could be altered by the redox reaction. Furthermore, the figures of merit of three complexes were evaluated for nonlinear photonic applications in optical limiter, saturable absorber, or all-optical switch.

Keywords: Phthalocyanine; Sandwich type; Rare earth; Nonlinear refraction; Nonlinear absorption; Femtosecond-pulsed Z-scan technique. 


\section{Introduction}

In the past decade, phthalocyanines (Pcs) and their derivatives have been extensively investigated because they have advantages of high thermal and chemical stability, large delocalized $\pi$-electron system, structural flexibility to host different central metal ions and various functionalizations at the peripheral positions [1-3]. Owing to the existing large metal ions, such as rare earths, actinides, some early transition metals, and main group elements, these macrocycles can form half-sandwich structure [4], sandwich-type double-decker structure [5], sandwich-type triple-decker structure [6], and binuclear phthalocyanine-containing sandwich structure [7]. Usually, the sandwich-type bis(phthalocyaninato) rare earth complexes possess peculiar electrical and magnetic properties enabling them to be used as gas sensors [8,9], organic field effect transistors [10], molecular information storage materials [11], etc. More interestingly, these types of complexes exhibit the strong optical nonlinearities [2,12], which have broad nonlinear photonic applications in optical limiter, saturable absorber, all-optical switch, and so on. To protect human eyes and sensitive optical elements from laser-induced damage, an ideal optical limiter is expected to have high transmittance for low intensity inputs, while possessing lower transmittance for high intensity inputs. For the practical optical limiter, the material should have strong ultrafast nonlinear absorption, broadband spectral response, as well as low cost and ease of processing [13]. Saturable absorber, which has the strong saturable absorption signal with the fast response time and low saturable intensity, is extensively used in ultrashort-pulsed laser generations as crucial 
mode-locking and Q-switching elements [14]. For realization of all-optical switch, nonlinear optical materials should meet the requirements of large nonlinear refraction, low absorption losses due to both linear and nonlinear absorptions (high figures of merit), and ultrafast response times [15].

It is well documented that the enhancement of the third-order optical nonlinearities of bis(phthalocyaninato) rare earth complexes mainly originates from the introduction of rare earth metal ions with its large $\pi$-conjugated system and strong intramolecular $\pi-\pi$ interaction [2,12]. By appropriate design of macrocycles, researchers have established various sandwich-type design strategies, including the double- or triple-decker molecular structure $[5,6]$, the multiple-decker structure [16], and the mixed triple-decker structure [12]. At the same time, many efforts are concentrated on elucidating structure-property relationships for the optical refractive and absorptive nonlinearities of sandwich-type rare-earth multiple-decker complexes. It is noteworthy that the optical nonlinearities of materials could be manipulated by the variation of the rare earth atomic radius of complexes [17], the excitation optical intensity [18], the introduction of the metal cations of complexes [19], the annealing process of metallophthalocyanine thin films [20,21], the different peripheral substituents [22], the number of branches of oligothienylenevinylenes [23], as well as the size of nanoparticles [24].

During the past few decades, the redox reaction of bis(phthalocyaninato) rare earth complexes has been investigated. For examples, Pondaven et. al. [25] obtained satisfactory nuclear magnetic resonance spectra by adding hydrazine hydrate ( $1 \% \mathrm{~V} / \mathrm{V})$ 
to solutions of lutetium sandwiches in deuterated dimethylformamide. Shirk et. al. [26] reported the third-order optical nonlinearities of the neutral metallobis(phthalocyaninato) and their anions by degenerate four-wave mixing at $1.064 \mu \mathrm{m}$. Zhang et. al. [27] studied the redox behavior of the first slipped pseudo-quadruple-decker complex of phthalocyanines by cyclic voltammetry and differential pulse voltammetry in $\mathrm{CH}_{2} \mathrm{Cl}_{2}$. All these redox processes can be attributed to the successive removal or addition of electrons from or to the ligand-based orbitals of the macrocycles, whereas the centered trivalent lanthanide cannot be oxidized or reduced under the redox reaction [28]. Obviously, one may expect that the appearance of novel optical nonlinearities in the oxidized and reduced species of sandwich-type bis(phthalocyaninato) rare earth complexes, although the related investigations are seldom in the literature.

In this work, we prepared homoleptic bis(phthalocyaninato) rare earth double-decker complexes $\operatorname{Pr}(\mathrm{OOPc})_{2}$ [H ${ }_{2} \mathrm{OOPc}=2,3,9,10,16,17,23,24-$ octakis(octyloxy)phthalocyanine] (1), the oxidized $\left[\operatorname{Pr}(\mathrm{OOPc})_{2}\right]^{+}(2)$, and the reduced $\left[\operatorname{Pr}(\mathrm{OOPc})_{2}\right]^{-}$(3). We systematically investigated the nonlinear optical properties of these complexes by performing the femtosecond-pulsed Z-scan experiments at the wavelength of $800 \mathrm{~nm}$. We found that the second-order hyperpolarizability $\gamma_{R}$ in complexes decrease in the order of $\mathbf{1}>\mathbf{2}>\mathbf{3}$. Particularly, the observed nonlinear absorption processes alter from two-photon absorption (2PA) in complex 2 to saturable absorption in complex 3. The mechanism of the optical nonlinearity was discussed in detail. The results verified that the 
nonlinear optical properties of bis(phthalocyaninato) rare earth complexes could be manipulated by the redox reaction. Furthermore, we evaluated the figures of merit (FOMs) of these complexes for nonlinear photonic applications in optical limiter, saturable absorber, or all-optical switch.

\section{Experimental section}

\subsection{Material preparation and characterization}

The homoleptic substituted bis(phthalocyaninato) rare earth double-decker complex $\operatorname{Pr}(\mathrm{OOPc})_{2}\left[\mathrm{H}_{2} \mathrm{OOPc}=2,3,9,10,16,17,23,24\right.$-octakis(octyloxy)phthalocyanine] (1) was synthesized according to previously reported methods $[8,9,29]$. The synthesized procedure of three complexes is shown in Fig. 1(a). The detailed synthesis and characterization of complex $\operatorname{Pr}(\mathrm{OOPc})_{2}$ can be found elsewhere $[8,9,29]$. A part of complex 1 dissolved in highly soluble chloroform-methanol (2:1, V/V) was divided two parts for the redox reaction. One part was added iodine $(0.1 \mathrm{mg} / \mathrm{mL})$ and the oxidized $\left[\operatorname{Pr}(\mathrm{OOPc})_{2}\right]^{+}$(2) with two radical anions was obtained. Another part was added hydrazine hydrate $(5 \mathrm{uL} / \mathrm{mL}$ ) as a reducing agent to determine the monoanion $\left[\mathrm{Pr}(\mathrm{OOPc})_{2}\right]^{-}(3)$ in which both macrocycles are dianionic. The molecular structures of three complexes 1-3 are shown in Fig. 1(b).

Following the reported work by Zhu et. al. [28], the electrochemistry of complex 1 was studied by cyclic voltammetry and differential pulse voltammetry in $\mathrm{CH}_{2} \mathrm{Cl}_{2}$. The complex 1 underwent one quasi-reversible one-electron oxidation and three quasi-reversible one-electron reductions in the electrochemical window. Compared 
the potential difference between the first oxidation and first reduction processes for complex 1, 0.41V, with its unsubstituted bis(phthalocyaninato) double decker complex $\operatorname{Pr}(\mathrm{Pc})_{2}, 0.36 \mathrm{~V}$, we concluded that complex 1 has effective $\pi-\pi$ interaction between the two phthalocyanine ligands.

\subsection{IR spectra}

As is well known, IR spectroscopy is a useful tool for analyzing the structure properties of the sandwich-type bis(phthalocyaninato) rare earth complexes [29,30,31]. Figure 2 shows the IR spectrum of complex 1 in the range of fundamental frequencies 400-4000 $\mathrm{cm}^{-1}$, which was recorded in $\mathrm{KBr}$ pellet with $0.09 \mathrm{~cm}^{-1}$ resolution using a NEXUS670 spectrometer. For the sake of comparison, characteristic IR bands of the complex $\operatorname{Pr}(\mathrm{OOPc})_{2}$ reported previously [31] are also shown by the circles in Fig. 2. Clearly, our results are in good agreement with the reported ones, implying that we indeed synthesized the complex $\mathbf{1}$. Vibrational frequencies in the spectral region from 400 to $1000 \mathrm{~cm}^{-1}$ are derived from the aromatic inplane C-H stretch. The complex $\mathbf{1}$ shows the C-O-C stretching bands at $1047 \mathrm{~cm}^{-1}$ (symmetric) and $1277 \mathrm{~cm}^{-1}$ (anti symmetric). The phthalocyanine $\pi$-radical marker band was observed at $1315 \mathrm{~cm}^{-1}$. In addition, the intense bands at $2850-2960 \mathrm{~cm}^{-1}$ are contributed to the C-H stretching vibrations of the octyloxy side chains.

\subsection{Electronic absorption spectra}

The double-deckers 1-3 were respectively dissolved in chloroform-methanol (2:1, $\mathrm{V} / \mathrm{V}$ ) with the concentration of $1 \times 10^{-4} \mathrm{M}$ and contained in 1-mm-thick quartz cells for optical spectra measurements. The UV-vis-IR linear absorption spectra of three 
complexes in solutions, as shown in Fig. 3, were recorded at room temperature with a spectrophotometer (Shimadzu UV-3600). Besides, the linear absorption spectrum of a neat solvent of chloroform-methanol $(2: 1, \mathrm{~V} / \mathrm{V})$ was measured for reference and no absorption band was observable. Consequently, it is concluded that the absorption of the solutions in the $300-800 \mathrm{~nm}$ range originate from the complexes only. The linear absorption coefficients $\alpha_{0}$ were evaluated from the linear transmission spectra of the solutions after correction of reflection losses at the air/sample and sample/air interfaces. The measured values of $\alpha_{0}$ at $800 \mathrm{~nm}$ are listed in Table 1.

As shown the solid line in Fig. 3, the linear absorption spectrum of complex $\operatorname{Pr}(\mathrm{OOPc})_{2}$ (1) has typical phthalocyanine features of the splitting Soret bands with maxima at $341-373 \mathrm{~nm}, \pi$-radical anion band at $509 \mathrm{~nm}$, and the main Q band at 600-691 nm. Compared with the linear absorption spectrum of complex 1, the $\pi$-radical anion band of the oxidized species $\left[\operatorname{Pr}(\mathrm{OOPc})_{2}\right]^{+}(2)$ is red-shifted to 532 $\mathrm{nm}$ and the absorption of Soret bands is enhanced. For the linear absorption spectrum of the complex $\left[\operatorname{Pr}(\mathrm{OOPc})_{2}\right]^{-}$(3), as displayed the dotted line in Fig. 3, significant blue-shift takes place on the Q band to be $648 \mathrm{~nm}$ and the Soret band appears at 355 nm as an intense band, while the $\pi$-radical anion band disappears. The variations of the linear absorption spectra of complexes 2 and $\mathbf{3}$ are due to the changes of $\pi-\pi$ electron transitions and $n-\pi$ electron transitions by the redox reaction [32]. It is noteworthy that all these complexes presented here have the similar linear absorption spectra with other homoleptic substituted bis(phthalocyaninato) rare earth double-decker complexes [17,33]. 


\subsection{Nonlinear optical characterization technique}

The nonlinear optical properties of complexes were investigated by a single-beam Z-scan technique [34]. The complexes in chloroform-methanol (2:1, V/V) with the concentration of $5 \times 10^{-4} \mathrm{M}$ were contained in 1-mm-thick quartz cells for the measurements. It is desirable to choose the solvent with negligible nonlinear optical effect for the estimation of optical nonlinearities of complexes. However, most of the organic solvents exhibit the third-order refractive nonlinearity under the excitation of sub-picosecond laser pulses [35]. Nevertheless, the nonlinear refraction contribution of both the solvent and solute can be separated, according to the related theory [36,37]. Accordingly, we chose the chloroform-methanol $(2: 1, \mathrm{~V} / \mathrm{V})$ as a solvent due to the fact that complexes are highly soluble in this solvent.

In the Z-scan experiments, the laser source was a Ti:sapphire regenerative amplifier (Coherent Inc.), operating at a wavelength of $800 \mathrm{~nm}$ with a pulse duration of $170 \mathrm{fs}$, a repetition rate of $1 \mathrm{kHz}$, and a near-Gaussian temporal and spatial profiles. The laser beam was focused by an achromatic lens with a focal length of $150 \mathrm{~mm}$, producing the beam waist at the focus $\omega_{0}=14.3 \mu \mathrm{m}$. To carry out Z-scans, the sample was scanned across the focus along the $z$-axis using a computer-controlled translation stage, while the transmitted pulse energies in the presence or absence of the far-field aperture were probed by a detector, obtaining the closed- and open-aperture Z-scans, respectively. The linear aperture transmittance of the far-field aperture was measured to be $S=0.3$ in the closed-aperture Z-scan configuration. Besides, the Z-scan measurement system was calibrated by a piece of cadmium 
sulfide bulk crystal. The result indicates that the experimental uncertainty was within $\pm 10 \%$.

\section{Results and discussion}

To obtain the nonlinear optical coefficients and to gain an insight on the physical mechanisms for the observed optical nonlinearities, we performed the closed- and open-aperture Z-scan experiments at different levels of laser intensities $I_{0}$. Besides, to exclude the optical nonlinearity arising from the solvent, we also conducted Z-scan experiments on the chloroform-methanol $(2: 1, \mathrm{~V} / \mathrm{V})$ in the quartz cell. Inasmuch as all the measured closed- and open-aperture Z-scans at different intensities are similar in configuration. As an example, Fig. 4 shows Z-scans for the pure solvent and the solutions of complexes 1-3 at $I_{0}=173 \mathrm{GW} / \mathrm{cm}^{2}$. The closed-aperture Z-scans show salient features as follows: (i) the symmetric valley-to-peak configuration for pure solvent displayed by the circles in Fig. 4(a); (ii) an enhanced valley and a suppressed peak for both 1 and 2 (see Fig. 4(b) and 4(c)); and (iii) a suppressed valley and an enhanced peak for 3 (see Fig. 4(d)). Such characteristics of valley-to-peak configurations in the closed-aperture Z-scans indicate the presence of the positive nonlinear refraction effect for all samples. For the pure solvent, as shown the squares in Fig. 4(a), the nonlinear absorption effect can be safely ignored because no peak or valley was detected in the open-aperture Z-scans at the intensity less than 230

GW/cm². In Fig. 4(b) and 4(c), however, the open-aperture Z-scans display a symmetric valley with respect to the focus, suggesting that both $\mathbf{1}$ and $\mathbf{2}$ exhibit the 
positive nonlinear absorption in nature. Interestingly, the open-aperture Z-scan shown in Fig. 4(d) has a peak configuration with respect to the focus, implying that the saturable absorption plays a primary role for the nonlinear absorption properties of $\mathbf{3}$. Apparently, the observed nonlinear absorption originates from the solute only. More importantly, the nonlinear absorption properties of complex solutions are manipulated by the redox reaction.

Under the assumption that the solutions of complexes $\mathbf{1}$ and $\mathbf{2}$ only possess the third-order optical nonlinearities, we evaluated the nonlinear absorption coefficient $\alpha_{2}$ and nonlinear refraction index $n_{2}$ from the best fitting between the femtosecond-pulsed Z-scan theory [38] and the measured Z-scans at different levels of $I_{0}$. Note that we adopted the analytical formulas of the Z-scan technique for extracting the nonlinear refraction index from the closed-aperture Z-scan trace, instead of the so-called "divided” closed-aperture Z-scans [34,39,40]. This is because that our experimental results (see Fig. 4(b) and 4(c)) indicate the strong nonlinear absorption and the relative small nonlinear refraction. In this case, the relative error of the obtained nonlinear refraction index given by the division method could exceed over $50 \%$ [41]. For the pure solvent of chloroform-methanol (2:1, V/V), the obtained values are $\alpha_{2}=0$ and $n_{2}^{\text {solvent }}=2.8 \times 10^{-7} \mathrm{~cm}^{2} / \mathrm{GW}$, which are comparable to the ones for organic solvents [35]. We found that the extracted values of $\alpha_{2}$ and $n_{2}$ for complexes 1 and $\mathbf{2}$ are independent of $I_{0}$, which confirm that samples indeed exhibit the third-order optical nonlinearities in nature. As an example, Figure 5 illustrates the measured $\alpha_{2}$ values as a function of $I_{0}$ for the chloroform-methanol solutions of 
complexes $\mathbf{1}$ and $\mathbf{2}$. It is noted that the complex $\mathbf{3}$ exhibits the saturable absorption behavior and the positive nonlinear refraction effect, as shown in Fig. 4(d). Consequently, the photoinduced absorption and refraction can be described by $\Delta \alpha=\alpha_{0} /\left(I+I_{S}\right)$ and $\Delta n=n_{2} I \quad$ [42], where $I_{S}$ is the saturable intensity. By fitting to the Z-scan theory on a saturable absorber [42], the saturable intensity of complex $\mathbf{3}$ was picked up to be $I_{S}=40 \mathrm{GW} / \mathrm{cm}^{2}$ with the known parameters $\alpha_{0} L=0.42$ and $I_{0}=173 \mathrm{GW} / \mathrm{cm}^{2}$. In addition, all the Z-scans measured at the other different levels of intensities from 100 to $230 \mathrm{GW} / \mathrm{cm}^{2}$ can also be reproduced by the Z-scan theory on a saturable absorber. Besides, the measured $I_{S}$ and $n_{2}$ are independent of the optical intensity $I_{0}$, indicating that the observed nonlinear absorption and refraction of $\mathbf{3}$ are only a saturable absorption process and the third-order nonlinear refraction, respectively. The measured nonlinear parameters for three complex solutions at 800 $\mathrm{nm}$ are summarized in Table 1 . We numerically simulated the Z-scans using the measured parameters $\left(\alpha_{2}, n_{2}\right.$, or $\left.I_{S}\right)$ as displayed by the solid lines in Fig. 4, confirming the validity of our measurements.

To estimate the refractive nonlinearity of the solute, we assume a solution of non-interacting particles. Accordingly, the effective refractive nonlinearity in a pairwise additive model is expressed by $n_{2}^{\text {solution }}=f n_{2}^{\text {solute }}+(1-f) n_{2}^{\text {solvent }}$. Here $f$ is the dilute solution containing a weight fraction of solute, $n_{2}^{\text {solute }}$ and $n_{2}^{\text {solvent }}$ are the nonlinear refraction indices of solute and solvent, respectively [36,37]. With the known parameters of $n_{2}^{\text {solvent }}=2.8 \times 10^{-7} \mathrm{~cm}^{2} / \mathrm{GW}$ and $f=0.13 \%$, we determined the intrinsic nonlinear refraction indices for complexes $\mathbf{1}, \mathbf{2}$, and $\mathbf{3}$ to be 
$n_{2}^{\text {solute }}=7.7 \times 10^{-5}, 3.9 \times 10^{-5}$, and $3.1 \times 10^{-5} \mathrm{~cm}^{2} / \mathrm{GW}$, respectively. Because the concentration of the complexes in solution is extremely dilute $\left(5 \times 10^{-4} \mathrm{M}\right.$, alternatively, $f=0.13 \%$ ), the estimated nonlinear refraction indexes of three solutes are about two orders of magnitude larger than those of both the solution and solvent.

In general, the molecular second-order hyperpolarizability $\gamma_{R}$ is introduced to appraise the contribution of one molecule to the refractive nonlinearity. The $\gamma_{R}$ value is calculated through the relation $\gamma_{R}=n_{0}^{2} \varepsilon_{0} c n_{2}^{\text {solute }} /\left(N L^{4}\right)$, where $L$ is the local field factor expressed by $L=\left(n_{0}^{2}+2\right) / 3, n_{0}$ is the linear refractive index of solution, $N$ is the density of molecules in the unit of number of molecules per $\mathrm{cm}^{3}, \varepsilon_{0}$ is the permittivity of vacuum, and $c$ is the velocity of light in vacuum [36]. By using $N=3.0 \times 10^{17} \mathrm{~cm}^{-3}$ in our experiments, the calculated values of $\gamma_{R}$ at $800 \mathrm{~nm}$ for complexes are listed in Table 2. These $\gamma_{R}$ values are comparable to the ones reported by Chen et al. [43] for porphyrin-appended europium(III) bis(phthalocyaninato) complexes. Moreover, the $\gamma_{R}$ values of these complexes are about two orders of magnitude larger than those of the traditional mental phthalocyanines [44].

The linear absorption coefficient $\alpha_{0}$ is related to the absorptive cross section of the ground state $\sigma_{0}$ via $\alpha_{0}=\sigma_{0} N$. For the 2PA process, the 2PA coefficient $\alpha_{2}$ and 2PA cross section $\sigma_{2 \mathrm{PA}}$ are related through the conversion formula $\alpha_{2}=\sigma_{2 \mathrm{PA}} N /(\hbar \omega)$, where $\hbar \omega$ is the incident photon energy. Hence, the obtained values of $\sigma_{2 \mathrm{PA}}$ for complexes $\mathbf{1}$ and $\mathbf{2}$ at $800 \mathrm{~nm}$ are summarized in Table 2. The $\sigma_{2 \mathrm{PA}}$ values of these complexes are comparable with those of sandwich-type mixed (phthalocyaninato)(porphyrinato) europium double- and triple-decker complexes [2]. 
For complex 3, the saturable absorption effect is well described by a simple two-level system (i.e., a ground state $S_{0}$ and an excited state $S_{1}$ [42]). As shown in Fig. 4(d), the strong saturable absorption and no reverse saturable absorption implies that the absorption cross section of $S_{0}$ is larger than the absorption cross section of $S_{1}$. Consequently, we determined the absorption cross section of $S_{1}$ to be $\sigma_{S_{1}}=1.2 \times 10^{-17}$ $\mathrm{cm}^{-2}$, according to the conversion formula $T / T_{\text {lin }}=1-\alpha_{0} \sigma_{S_{1}} F_{0} L_{\text {eff }} /(4 \hbar \omega)$, where $T$ and $T_{\text {lin }}$ are respectively the nonlinear and linear transmittances, $F_{0}$ is the fluence of the beam at the focus, and $L_{\text {eff }}$ is the effective length of the sample [45].

From Table 2, one easily finds that the intrinsic refractive nonlinearity originating from a molecule (i.e., $\gamma_{R}$ ) decreases in the order of $\mathbf{1}>\mathbf{2}>\mathbf{3}$. Analogously, the nonlinear absorption behavior has the same trend. The nominal nonlinear absorption coefficient decreases from positive to negative because the nonlinear absorption changes from TPA to saturable absorption. It is well known that several factors could enhance the optical nonlinearity of sandwich-type bis(phthalocyaninato) rare earth complexes, such as the expanded $\pi$-electron system, intramolecular charge transfer, and heavy central metal ion [43]. For the molecular structure of $\operatorname{Pr}(\mathrm{OOPc})_{2}$ (1) illustrated in Fig. 1(b), the central metal praseodymium ion (+3) coordinates to two phthalocyanine ligands with an unpaired electron residing on them, suggesting that the neutral complexes is mixed-valence compound [26]. Such a intervalence complex has a characteristic electronic transition in the near-IR region to provide a new degree of freedom for charge redistribution under the excitation of intense laser pulses, resulting in the huge enhancement of the third-order nonlinear optical properties of 
bis(phthalocyaninato) rare earth complexes. The oxidized $\left[\operatorname{Pr}(\mathrm{OOPc})_{2}\right]^{+}(2)$ with two unpaired electrons delocalized over two phthalocyanine rings separately decreases the intramolecular charge transfer by reason of the symmetric structure. The reduced $\left[\mathrm{Pr}(\mathrm{OOPc})_{2}\right]^{-}$(3) without unpaired electron has no intervalence charge transfer transitions. On the other hand, the linear absorption spectrum of complex $\mathbf{3}$ with the intense Soret bands and Q band as well as no $\pi$-radical anion band suggests that the electronic transition induced by the reduced reaction is quietly different from those of neutral complexes and reduced species (see Fig. 3). As a result, the nonlinear optical responses of $\mathbf{1}$ and $\mathbf{2}$ are much larger than that of $\mathbf{3}$. In particular, the complex $\mathbf{3}$ exhibits the saturable absorption, while the complexes 1 and 2 have the 2PA effect. In a word, it is demonstrated that the inhomogenous distribution of electrons among the phthalocyanine macrocycles impacts on the third-order nonlinear properties of sandwich-type complexes.

Finally, we exploited the nonlinear optical properties of three complexes for nonlinear photonic applications. Since homoleptic substituted bis(phthalocyaninato) rare earth double-decker complexes $\mathbf{1}$ and $\mathbf{2}$ exhibit large 2PA effect at the wavelength of $800 \mathrm{~nm}$, we exploited their optical limiting applications. The optical limiting experiments, which were carried out by changing the input pulse energies and by monitoring both the input and the transmitted pulse energies simultaneously, were performed with the same experimental system used for the open-aperture Z-scan except that the sample was fixed at the focus. Figure 6 shows the intensity-dependent transmissions of three complexes in chloroform-methanol solutions with the 
concentration of $5.0 \times 10^{-4} \mathrm{M}$. It can be seen that the normalized transmittances for three complexes are a constant until the input intensity of $\sim 10 \mathrm{GW} / \mathrm{cm}^{2}$. However, when the input intensity increases beyond $\sim 10 \mathrm{GW} / \mathrm{cm}^{2}$, the measured transmittance deviates from the linearity and has notable features as follows: (i) dramatically decreasing at $\sim 50 \mathrm{GW} / \mathrm{cm}^{2}$ for both 1 and 2, which suggests the occurrence of optical limiting behavior; and (ii) synchronously increasing for $\mathbf{3}$, exhibiting the characteristic of the saturable absorption. Besides, the optical limiting ability of $\mathbf{1}$ is better than that of 2. In Fig. 6, the solid lines are numerically simulated with the known parameters obtained from Z-scan experiments, as listed in Table 1. Clearly, the experimental results are in good agreement with the numerical simulations. The results demonstrate that homoleptic substituted bis(phthalocyaninato) rare earth double-decker complexes with large 2PA effect exhibit a better optical limiting performance. On the contrary, the complex $\mathbf{3}$ exhibits the saturable absorption, which has potential applications in the ultrafast laser generations as passive mode-locked lasers and Q-switching elements. For saturable absorbing applications, the FOM is defined as $F O M=\sigma_{S_{1}} / \sigma_{0}$ [46]. The measured value of $F O M=0.86$ for complex 3 is comparable with that of 0.72 for $\mathrm{Sb}_{2} \mathrm{Se}_{3}$ nanowires [47], which is close to the target value of $F O M<1$ [46]. As summarized in Table 1, the complexes 1 and 2 with large nonlinear refraction and low nonlinear absorption can be exploited for all-optical switching. To evaluate the requirements for all-optical switching, we calculated two FOMs (i. e., $W$ and $T$ ). Here $W=n_{2} I /\left(\alpha_{0} \lambda\right)$ and $T=\alpha_{2} \lambda / n_{2}$ are the one- and two-photon FOMs, respectively [37,48]. Taking the intensity $I=150 \mathrm{GW} / \mathrm{cm}^{2}$ for 
the calculation of $W$, we obtain $W=90$ and $T=5.0 \times 10^{-2}$ for complex 1 and $W=33$ and $T=6.6 \times 10^{-2}$ for complex 2 , which are close to the target values of $W>1$ and $T<1 \quad[37,48]$.

\section{Conclusion}

In summary, we prepared homoleptic bis(phthalocyaninato) rare earth double-decker complex $\operatorname{Pr}(\mathrm{OOPc})_{2}(\mathbf{1})$, and its oxidized species $\left[\operatorname{Pr}(\mathrm{OOPc})_{2}\right]^{+}(\mathbf{2})$ and reduced species $\left[\operatorname{Pr}(\mathrm{OOPc})_{2}\right]^{-}$(3) by adding iodine or hydrazine hydrate to $\mathbf{1}$, respectively. By performing the closed- and open-aperture Z-scan experiments with 170 fs laser pulses in the near infrared region at different levels of laser intensities, we determined both the third-order nonlinear refraction index and nonlinear absorption coefficient of three complexes. It is found that the second-order hyperpolarizability in complex molecules decreases in the order of $\mathbf{1}>\mathbf{2}>\mathbf{3}$. Furthermore, the nonlinear absorption effect changes from 2PA in complex 2 to saturable absorption in complex 3. The corresponding mechanism of the observed optical nonlinearities was discussed in detail. The results demonstrate that the nonlinear optical properties of sandwich-type bis(phthalocyaninato) rare earth complexes could be altered by the redox reaction. Furthermore, the FOMs with ultrafast response suggest that the homoleptic bis(phthalocyaninato) rare earth double-decker complex and its redox species should be desirable for nonlinear photonic applications in optical limiter, saturable absorber, or all-optical switch. 


\section{Acknowledgements}

This work was supported by the National Science Foundation of China (Grant Nos: 11474052, 11504049, 11174160, 21401075) and National Key Basic Research Program of China (2015CB352002).

\section{References}

[1] Evren D, Yenilmez HY, Kalkan Burat A. Microwave-assisted synthesis of novel nonperipherally substituted metallophthalocyanines bearing (7-(trifluoromethyl)quinolin-4-yl)oxy groups. Turk J Chem 2014; 38: 1174-84.

[2] Sheng N, Yuan ZD, Wang JX, Chen WD, Sun J, Bian YZ. Third-order nonlinear optical properties of sandwich-type mixed (phthalocyaninato)(porphyrinato) europium double- and triple-decker complexes. Dyes Pigments 2012; 95: 627-31.

[3] Binnemans K, Sleven J, De Feyter S, De Schryver FC, Donnio B, Guillon D. Structure and mesomorphic behavior of alkoxy-substituted bis(phthalocyaninato) lanthanide (III) complexes. Chem Mater 2003; 15: 3930-8.

[4] Bienfait AM, Wolf BM, Törnroos KW, Anwander R. Ln(II)/Pb(II)-Ln(III)/Pb(0) redox approach toward rare-earth-metal half-sandwich complexes. Organometallics 2015; 34: 5734-44.

[5] Gao YN, Li RJ, Dong S, Bian YZ, Jiang JZ. Bis[1,4,8,11,15,18,22,25-octa(butyloxyl)phthalocyaninato] rare earth double-decker complexes: synthesis, spectroscopy, and molecular structure. Dalton Trans 2010; 39: 1321-7.

[6] Kan JL, Wang HL, Sun W, Cao W, Tao J, Jiang JZ. Sandwich-type mixed 
tetrapyrrole rare-earth triple-decker compounds. Effect of the coordination geometry on the single-molecule-magnet nature. Inorg Chem 2013; 52: 8505-10.

[7] Huang $\mathrm{CH}$, Wang $\mathrm{K}$, Sun JS, Jiang JZ. Planar binuclear phthalocyanine-containing sandwich-type rare-earth complexes: synthesis, spectroscopy, electrochemistry, and NLO properties. Eur J Inorg Chem 2014: 1546-51.

[8] Xie D, Jiang YD, Wei P, Jiang JZ, Wu ZM, Li YR. Study on bis[phthalocyaninato] praseodymium complex/silicon hybrid chemical field-effect transistor gas sensor. Thin Solid Films 2002; 406: 262-7.

[9] Xie D, Jiang YD. The properties of praseodymium bis[octakis(octyloxy)phthalocyaninato] complex Langmuir-Blodgett films for $\mathrm{NO}_{2}$ sensor. Sensors and Actuators B 2003; 93: 379-83.

[10] Chen Y, Su W, Bai M, Jiang JZ, Li XY, Liu YQ, et al. High performance organic field-effect transistors based on amphiphilic tris(phthalocyaninato) rare earth triple-decker complexes. J Am Chem Soc 2005; 127: 15700-1.

[11] Liu Z, Yasseri AA, Lindsey JS, Bocian DF. Molecular memories that survive silicon device processing and real-world operation. Science 2003; 302: 1543-5.

[12] Li ZG, Gao F, Xiao ZG, Ao GH, Wu XZ, Fang Y, et al. Synthesis and third-order nonlinear optical properties of a sandwich-type mixed (phthalocyaninato)(schiffbase) triple-decker complexes. Dyes Pigments 2015; 119: 70-4.

[13] Liu ZB, Tian JG, Guo Z, Ren DM, Du F, Zheng JY, et al. Enhanced optical limiting effects in porphyrin-covalently functionalized single-walled carbon 
nanotubes. Adv Mater 2008; 20: 511-5.

[14] Wu K, Zhang XY, Wang J, Li X, Chen JP. $\mathrm{WS}_{2}$ as a saturable absorber for ultrafast photonic applications of mode-locked and Q-switched lasers. Opt Express 2015; 23: 11453-61.

[15] Meng ZM, Qin F, Li ZY. Ultrafast all-optical switching in one-dimensional semiconductor-polymer hybrid nonlinear photonic crystals with relaxing Kerr nonlinearity. J Opt 2012; 14: 1013-20.

[16] Wang H, Qi D, Xie Z, Cao W, Wang K, et al. A sandwich-type phthalocyaninato metal sextuple-decker complex: synthesis and NLO properties. Chem Commun 2013; 49: 889-91.

[17] Sheng N, Liu DH, Gu B, He J, C YP. A series of homoleptic bis(phthalocyaninato) rare earth sandwich complexes with large two-photon absorption cross-section. Dyes Pigments 2015; 122: 346-50.

[18] Wang K, Long H, Fu M, Yang G, Lu PX. Intensity-dependent reversal of nonlinearity sign in a gold nanoparticle array. Opt Lett 2010; 35: 1560-2.

[19] Iliopoulos K, El-Ghayoury A, Ouazzani HE, Pranaitis M, Belhadj E, Ripaud E, et al. Nonlinear absorption reversing between an electroactive ligand and its metal complexes. Opt Express 2012; 20: 25311-6.

[20] Zawadzka A, Płóciennik P, Strzelecki J, Korcala A, Arof AK, Sahraoui B. Impact of annealing process on stacking orientations and second order nonlinear optical properties of metallophthalocyanine thin films and nanostructures. Dyes Pigments 2014; 101: 212-20. 
[21] Zawadzka A, Karakas A, Płóciennik P, Szatkowski J, Łukasiak Z, Kapceoglu A, et al. Optical and structural characterization of thin films containing metallophthalocyanine chlorides. Dyes Pigments 2015; 112: 116-26.

[22] Derkowska B, Wojdyła M, Bała W, Jaworowicz K, Karpierz M, Grote JG, et al. Influence of different peripheral substituents on the nonlinear optical properties of cobalt phthalocyanine core. J Appl Phys 2007; 101: 083112.

[23] Fuks-Janczarek I, Nunzi JM, Sahraoui B, Kityk IV, Berdowski J, Caminade AM, et al. Third-order nonlinear optical properties and two-photon absorption in branched oligothienylenevinylenes. Opt Commun 2002; 209: 461-6.

[24] Wang K, Long H, Fu M, Yang G, Lu PX. Size-related third-order optical nonlinearities of Au nanoparticle arrays. Opt Express 2010; 18: 13874-9.

[25] Pondaven A, Cozien Y, Lher M. Symmetrically and unsymmetrically substituted lutetium diphthalocyanines: synthesis and spectroscopic characterization. New J Chem 1992; 16: 711-8.

[26] Shirk JS, Lindle JR, Bartoli FJ, Boyle ME. Third-order optical nonlinearities of bis(phthalocyanines). J Phys Chem 1992; 96: 5847-52.

[27] Zhang HC, Wang RM, Zhu PH, Lai ZF, Han J, Choi CF, et al. The first slipped pseudo-quadruple-decker complex of phthalocyanines. Inorg Chem 2004; 43: 4740-2.

[28] Zhu PH, Lu FL, Pan N, Arnold DP, Zhang SY, Jiang JZ. Comparative electrochemical study of unsubstituted and substituted bis(phthalocyaninato) rare earth(III) complexes. Eur J Inorg Chem 2004; 3: 510-7. 
[29] Liu W, Jiang JZ, Du DM, Arnold DP. Synthesis and spectroscopic properties of homoleptic bis[octakis(octyloxy)phthalocyaninato] rare earth (III) sandwich complexes. Aust J Chem 2000; 53: 131-5.

[30] Jiang JZ, Bao M, Rintoul L, Arnold DP. Vibrational spectroscopy of phthalocyanine and naphthalocyanine in sandwich-type (na)phthalocyaninato and porphyrinato rare earth complexes. Coord Chem Rev 2006; 250: 424-48.

[31] Jiang JZ, Arnold DP, Yu H. Infra-red spectra of phthalocyanine and naphthalocyanine in sandwich-type (na)phthalocyaninato and porphyrinato rare earth complexes. Polyhedron 1999; 18: 2129-39.

[32] Li XL, Qi DD, Chen C, Yang LG, Sun JS, Wang HL, et al. Bis[octakis(2,6-dimethylphenoxy)phthalocyaninato] rare earth(III) complexes: structure, spectroscopic, and electrochemical properties. Dyes Pigments 2014; 101: 179-85.

[33] Jiang JZ, Liu RCW, Mak TCW, Dominic Chan TW, Ng DKP. Synthesis, spectroscopic and electrochemical properties of substituted bis(phthalocyaninato) lanthanide(III) complexes. Polyhedron 1997; 16: 515-20.

[34] Sheik-Bahae M, Said AA, Wei TH, Hagan DJ, Van Stryland EW. Sensitive measurement of optical nonlinearities using a single beam. IEEE J Quantum Electron 1990; 26: 760-9.

[35] Gong QH, Li JL, Zhang TT, Yang H. Ultrafast third-order optical nonlinearity of organic solvents investigated by subpicosecond transient optical Kerr effect. Chin Phys Lett 1998; 15: 30-1. 
[36] Zhao MT, Singh BP, Prasad PN. A systematic study of polarizability and microscopic third-order optical nonlinearity in thiophene oligomers. J Chem Phys 1988; 89: 5535-41.

[37] Samoc M, Samoc A, Lutherdavies B, Bao ZN, Yu LP, Hsieh B, et al. Femtosecond Z-scan and degenerate four-wave mixing measurements of real and imaginary parts of the third-order nonlinearity of soluble conjugated polymers. J Opt Soc Am B 1998; 15: 817-25.

[38] Gu B, Ji W, Huang XQ. Analytical expression for femtosecond-pulsed Z scans on instantaneous nonlinearity. Appl Opt 2008; 47: 1187-92.

[39] Kulyk B, Kerasidou AP, Soumahoro L, Moussallem C, Gohier F, Frère P, et al. Optimization and diagnostic of nonlinear optical features of $\pi$-conjugated benzodifuran-based derivatives. Rsc Adv 2016; 6: 14439-47.

[40] Guezguez I, Ayadi A, Ordon K, Iliopoulos K, Branzea DG, Migalska-Zalas A, et al. Zinc induced a dramatic enhancement of the nonlinear optical properties of an azo-based iminopyridine ligand. J Phys Chem C 2014; 118: 7545-53.

[41] Gu B, Huang XQ, Tan SQ, Wang HT. A precise data processing method for extracting $\chi^{(3)}$ from Z-scan technique. Opt Commun 2007; 277: 209-13.

[42] Gu B, Fan YX, Wang J, Chen J, Ding JP, Wang HT. Characterization of saturable absorbers using an open-aperture Gaussian-beam Z scan. Phys Rev A 2006; 73: 390-6.

[43] Chen L, Hu R, Xu J, Wang SQ, Li XY, Li SY, et al. Third-order nonlinear optical properties of a series of porphyrin-appended europium(III) bis(phthalocyaninato) 
complexes. Spectrochim Acta Mol Biomol Spectros 2013; 105: 577-81.

[44] Hou HW, Song YL, Xu H, Wei YL, Fan YT, Zhu Y, et al. Polymeric complexes with "piperazine-pyridine” building blocks: synthesis, network structures, and third-order nonlinear optical properties. Macromolecules 2003; 36: 999-1008.

[45] Wei TH, Hagan DJ, Sence MJ, Van Strylaud EW, Perry JW, Coulter DR. Direct measurements of nonlinear absorption and refraction in solutions of phthalocyanines. Appl Phys B 1992; 54: 46-51.

[46] Burshtein Z, Blau P, Kalisky Y, Shimony Y, Kokta MR. Excited-state absorption studies of $\mathrm{Cr}^{4+}$ ions in several garnet host crystals. IEEE J Quantum Electron 1998; 34: 292-9.

[47] Yadav RK, Sharma R, Aneesh J, Abhiramnath P, Adarsh KV. Saturable absorption in one-dimensional $\mathrm{Sb}_{2} \mathrm{Se}_{3}$ nanowires in the visible to near-infrared region. Opt Lett 2016; 41: 2049-52.

[48] Kang JU, Villeneuve A, Sheik-Bahae M, Stegeman GI, Al-hemyari K, Aitchison JS, et al. Limitation due to three-photon absorption on the useful spectral range for nonlinear optics in AlGaAs below half band gap. Appl Phys Lett 1994; 65: 147-9. 
Table 1 Measured linear absorption coefficient $\left(\alpha_{0}\right)$, 2PA coefficient $\left(\alpha_{2}\right)$, saturable intensity $\left(I_{S}\right)$, third-order nonlinear refraction index $\left(n_{2}\right)$ for three complex solutions under the excitation of femtosecond laser pulses at $800 \mathrm{~nm}$ (experimental uncertainty: $\pm 10 \%)$.

\begin{tabular}{ccccc}
\hline Complexes & $\alpha_{0}\left(\mathrm{~cm}^{-1}\right)$ & $\alpha_{2}(\mathrm{~cm} / \mathrm{GW})$ & $I_{S}\left(\mathrm{GW} / \mathrm{cm}^{2}\right)$ & $n_{2}\left(\mathrm{~cm}^{2} / \mathrm{GW}\right)$ \\
\hline $\operatorname{Pr}(\mathrm{OOPc})_{2}(\mathbf{1})$ & 1.6 & $4.8 \times 10^{-2}$ & $3.8 \times 10^{-7}$ \\
{$\left[\mathrm{Pr}(\mathrm{OOPc})_{2}\right]^{+}(\mathbf{2})$} & 2.2 & $3.2 \times 10^{-2}$ & & $3.3 \times 10^{-7}$ \\
{$\left[\mathrm{Pr}(\mathrm{OOPC})_{2}\right]^{-}(\mathbf{3})$} & 4.2 & 40 & $3.2 \times 10^{-7}$ \\
Solvent & & & $2.8 \times 10^{-7}$ \\
\hline
\end{tabular}


Table 2 Molecular intrinsic absorption cross section of the ground state $S_{0}\left(\sigma_{0}\right)$, absorption cross section of $S_{1}\left(\sigma_{S_{1}}\right)$, 2PA cross section ( $\left.\sigma_{2 \mathrm{PA}}\right)$, second-order hyperpolarizability $\left(\gamma_{R}\right)$ for complexes at $800 \mathrm{~nm}$ (experimental uncertainty: $\pm 10 \%$ ).

\begin{tabular}{ccccc}
\hline Complexes & $\sigma_{0}\left(\mathrm{~cm}^{2}\right)$ & $\sigma_{S_{1}}\left(\mathrm{~cm}^{2}\right)$ & $\sigma_{2 \mathrm{PA}}\left(\mathrm{cm}^{4} \cdot \mathrm{s} \cdot\right.$ photon $\left.^{-1}\right)$ & $\gamma_{R}(\mathrm{esu})$ \\
\hline $\operatorname{Pr}(\mathrm{OOPc})_{2}(\mathbf{1})$ & $5.3 \times 10^{-18}$ & $4.0 \times 10^{-47}$ & $4.4 \times 10^{-30}$ \\
{$\left[\operatorname{Pr}(\mathrm{OOPC})_{2}\right]^{+}(\mathbf{2})$} & $7.3 \times 10^{-18}$ & & $2.7 \times 10^{-47}$ & $2.2 \times 10^{-30}$ \\
{$\left[\operatorname{Pr}(\mathrm{OOPC})_{2}\right]^{-}(\mathbf{3})$} & $1.4 \times 10^{-17}$ & $1.2 \times 10^{-17}$ & & $1.8 \times 10^{-30}$ \\
\hline
\end{tabular}




\section{Figure Captions}

Fig. 1. (a) Synthetic procedure of rare earth complexes $\operatorname{Pr}(\mathrm{OOPc})_{2}(\mathbf{1}),\left[\operatorname{Pr}(\mathrm{OOPc})_{2}\right]^{+}$ (2), and $\left[\mathrm{Pr}(\mathrm{OOPc})_{2}\right]^{-}$(3). (b) Schematic molecular structure of three complexes.

Fig. 2. IR spectrum of complex 1 in the region of $400-4000 \mathrm{~cm}^{-1}$ recorded in $\mathrm{KBr}$ pellet with $0.09 \mathrm{~cm}^{-1}$ resolution. The circles are the characteristic IR bands of phthalocyanine for $\operatorname{Pr}(\mathrm{OOPc})_{2}$ from Ref. 31 .

Fig. 3. Linear absorption spectra of homolepticbis (phthalocyaninato) rare earth complexes $\operatorname{Pr}(\mathrm{OOPc})_{2} \quad(\mathbf{1}), \quad\left[\operatorname{Pr}(\mathrm{OOPc})_{2}\right]^{+} \quad(\mathbf{2}), \quad\left[\operatorname{Pr}(\mathrm{OOPc})_{2}\right]^{-} \quad$ (3) dissolved in chloroform-methanol $(2: 1, \mathrm{~V} / \mathrm{V})$ with the concentration of $1.0 \times 10^{-4} \mathrm{M}$. Inset is the photograph of the solutions of complexes.

Fig. 4. Examples of Z-scans measured at $I_{0}=173 \mathrm{GW} / \mathrm{cm}^{2}$ for (a) pure solvent of chloroform-methanol (2:1, V/V), (b) $\operatorname{Pr}(\mathrm{OOPc})_{2}$ solution (1), (c) $\left[\operatorname{Pr}(\mathrm{OOPc})_{2}\right]^{+}$ solution (2), and (d) $\left[\operatorname{Pr}(\mathrm{OOPc})_{2}\right]^{-}$solution (3). The circles and squares are the closedand open-aperture Z-scans, respectively; while the solid lines are the best-fit curves simulated by the femtosecond-pulsed Z-scan theory [38].

Fig. 5. Examples of intensity dependence of 2PA coefficient $\alpha_{2}$ for the chloroform-methanol solutions of complexes $\mathbf{1}$ and 2 . The scatters are the experimental results, while the dashed lines are for guidance to the eyes.

Fig. 6. Nonlinear transmission behaviors of the chloroform-methanol solutions of complexes with the concentration of $5.0 \times 10^{-4} \mathrm{M}$ as a function of the input irradiance under the femtosecond laser excitation at $800 \mathrm{~nm}$ wavelength. The scatters are the experimental results, while the solid lines are the theoretical simulations with 
the parameters obtained from Z-scan experiments. 
(a)

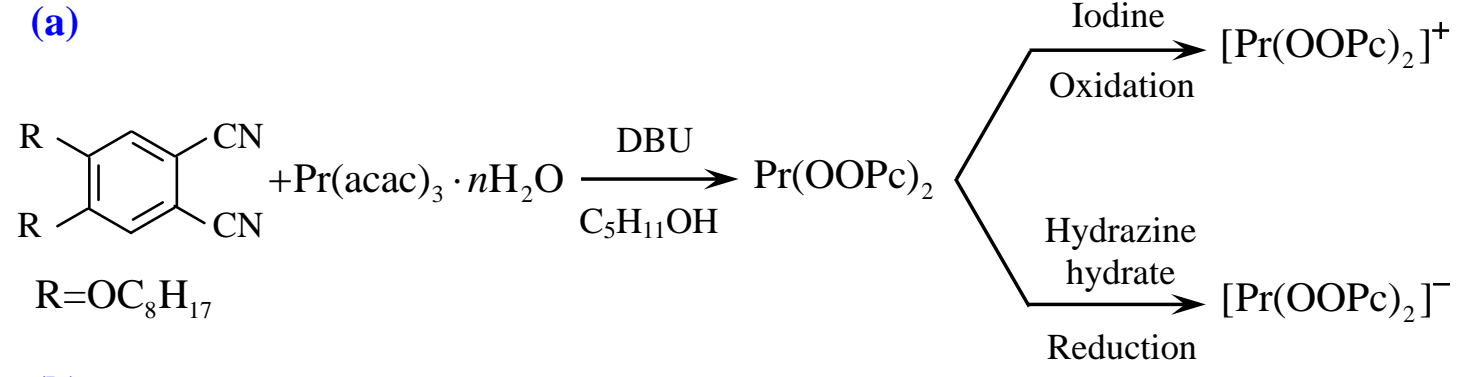

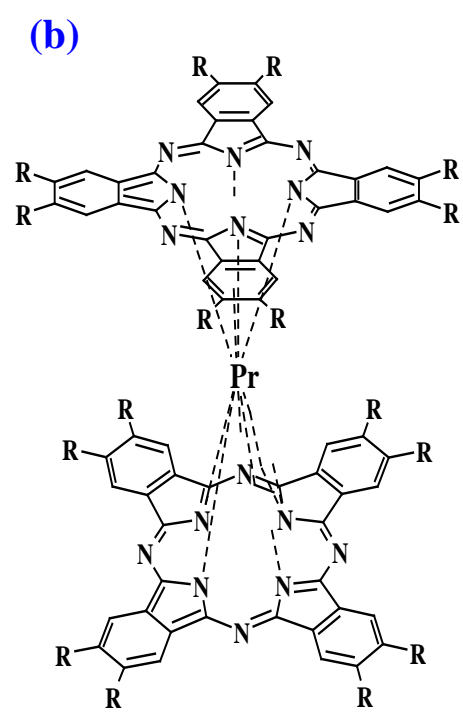

(1)

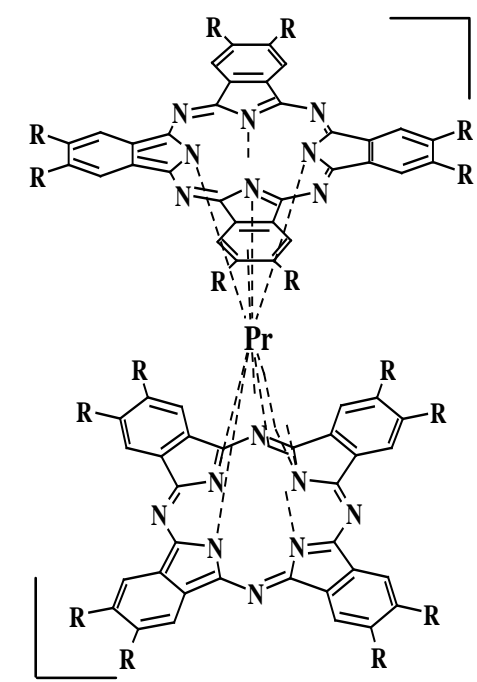

(2)

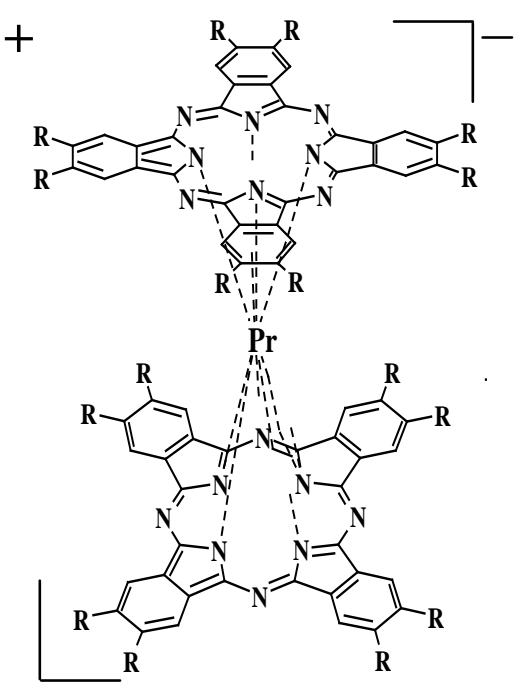

(3)

Fig. 1. (a) Synthetic procedure of rare earth complexes $\operatorname{Pr}(\mathrm{OOPc})_{2}(\mathbf{1}),\left[\operatorname{Pr}(\mathrm{OOPc})_{2}\right]^{+}$ (2), and $\left[\operatorname{Pr}(\mathrm{OOPc})_{2}\right]^{-}$(3). (b) Schematic molecular structure of three complexes. 


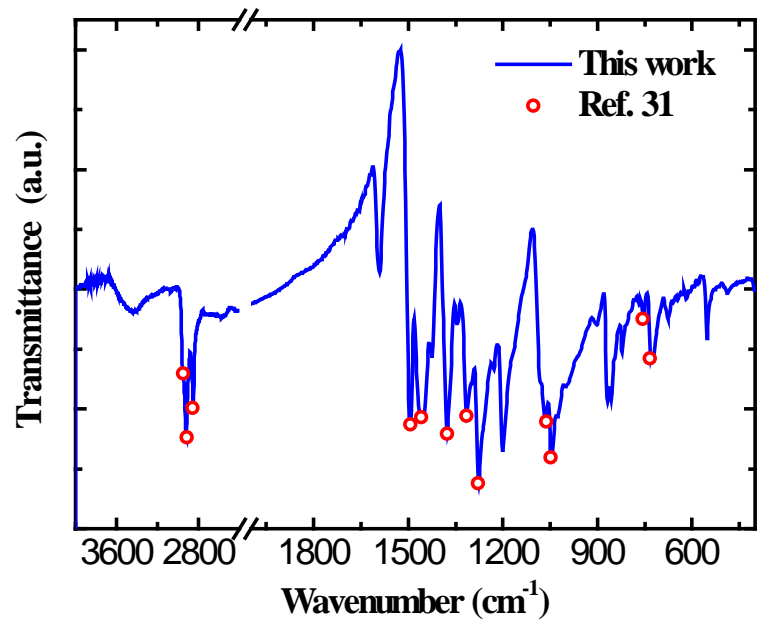

Fig. 2. IR spectrum of complex 1 in the region of $400-4000 \mathrm{~cm}^{-1}$ recorded in $\mathrm{KBr}$ pellet with $0.09 \mathrm{~cm}^{-1}$ resolution. The circles are the characteristic IR bands of phthalocyanine for $\operatorname{Pr}(\mathrm{OOPc})_{2}$ from Ref. 31 . 


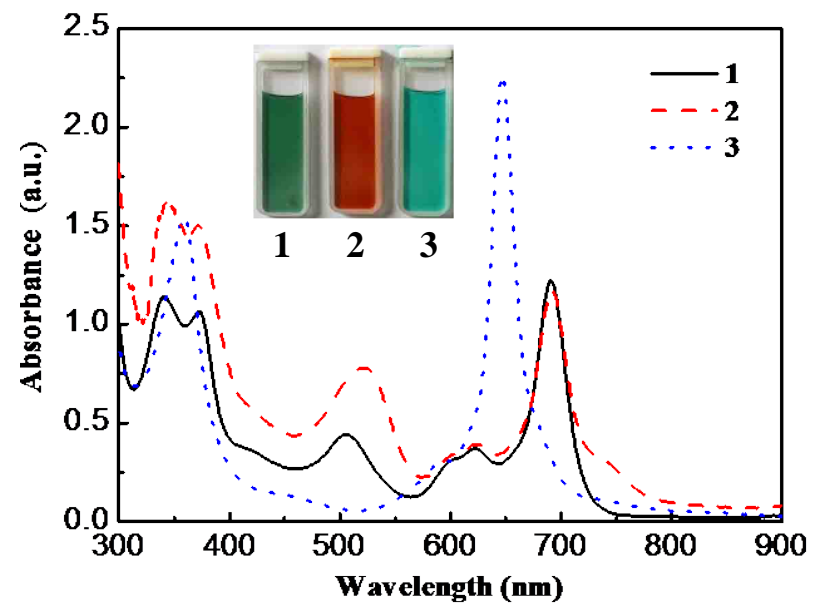

Fig. 3. Linear absorption spectra of homolepticbis (phthalocyaninato) rare earth complexes $\operatorname{Pr}(\mathrm{OOPc})_{2} \quad(\mathbf{1}), \quad\left[\operatorname{Pr}(\mathrm{OOPc})_{2}\right]^{+} \quad(\mathbf{2}), \quad\left[\operatorname{Pr}(\mathrm{OOPc})_{2}\right]^{-} \quad$ (3) dissolved in chloroform-methanol $(2: 1, \mathrm{~V} / \mathrm{V})$ with the concentration of $1.0 \times 10^{-4} \mathrm{M}$. Inset is the photograph of the solutions of complexes. 

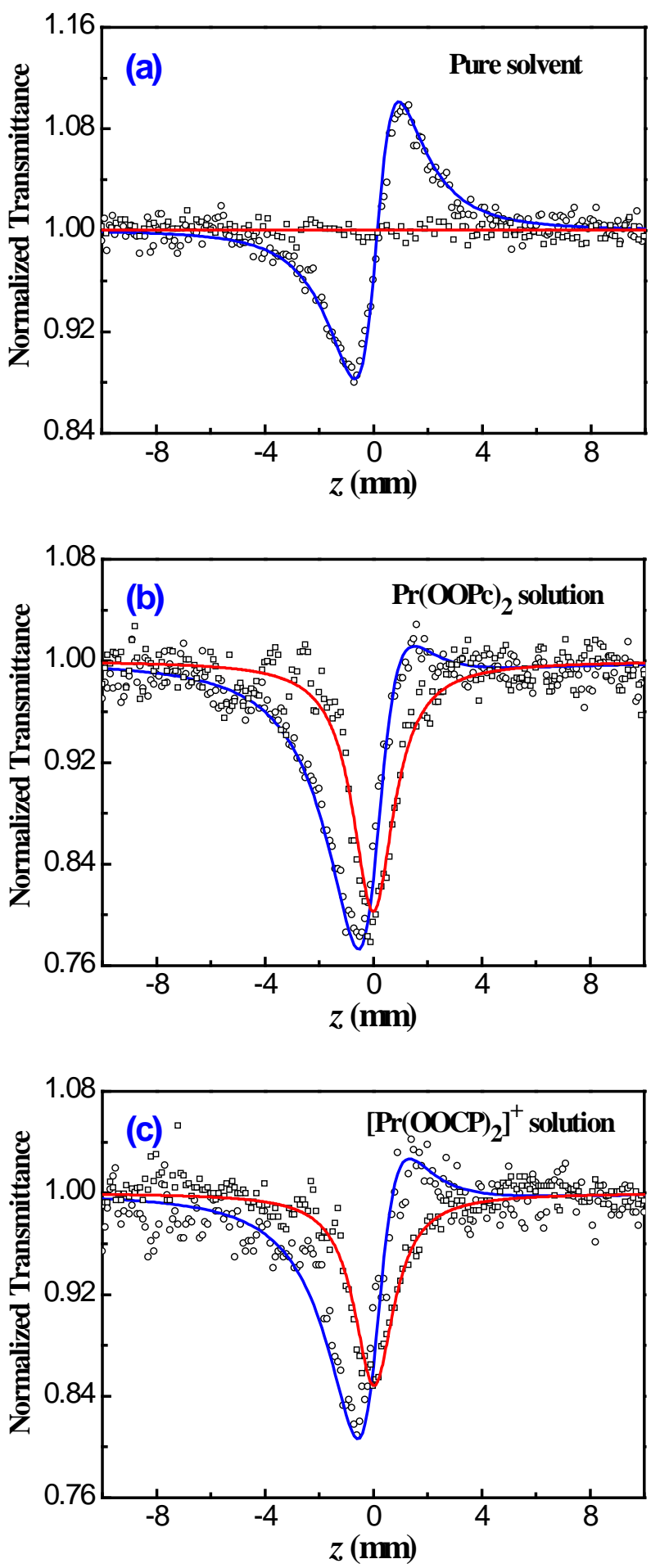


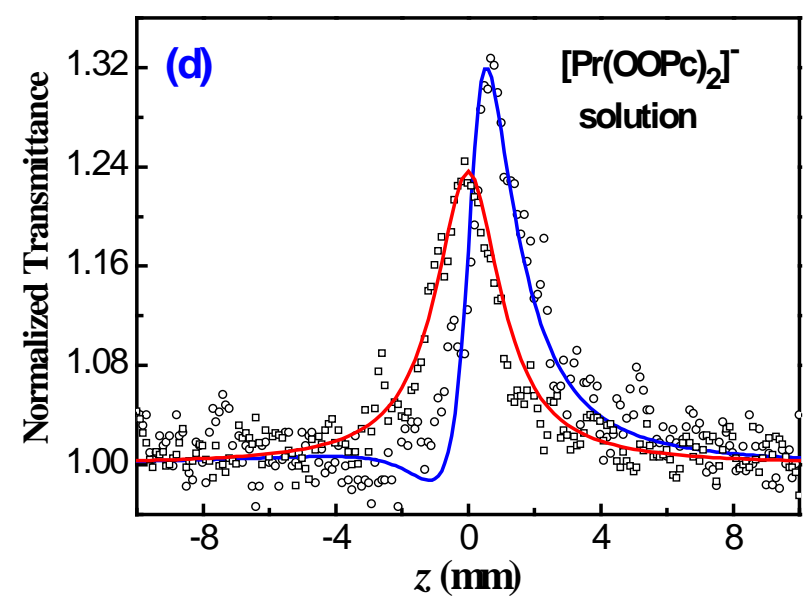

Fig. 4. Examples of Z-scans measured at $I_{0}=173 \mathrm{GW} / \mathrm{cm}^{2}$ for (a) pure solvent of chloroform-methanol (2:1, V/V), (b) $\operatorname{Pr}(\mathrm{OOPc})_{2}$ (1) solution, (c) $\left[\operatorname{Pr}(\mathrm{OOPc})_{2}\right]^{+}$(2) solution, and (d) $\left[\operatorname{Pr}(\mathrm{OOPc})_{2}\right]^{-}$(3) solution. The circles and squares are the closed- and open-aperture Z-scans, respectively; while the solid lines are the best-fit curves simulated by the femtosecond-pulsed Z-scan theory [38]. 


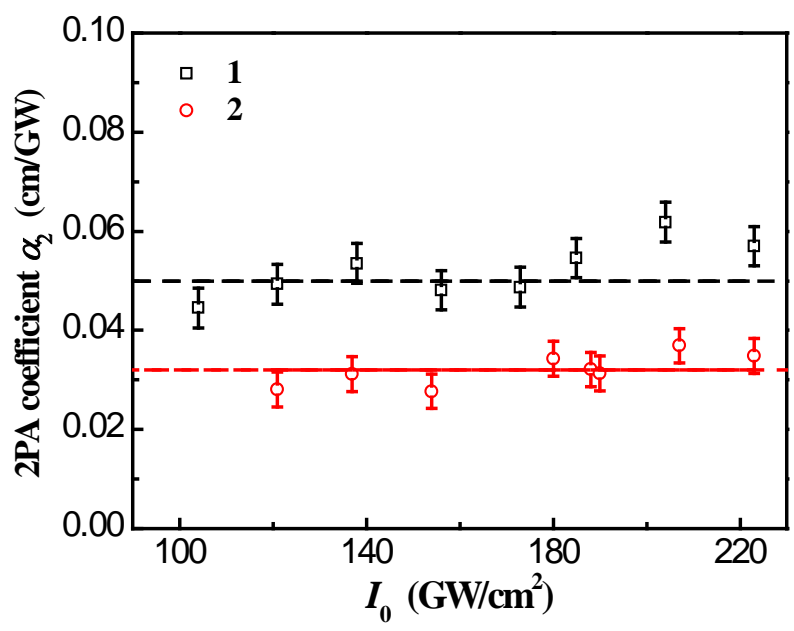

Fig. 5. Examples of intensity dependence of 2PA coefficient $\alpha_{2}$ for the chloroform-methanol solutions of complexes $\mathbf{1}$ and $\mathbf{2}$. The scatters are the experimental results, while the dashed lines are for guidance to the eyes. 


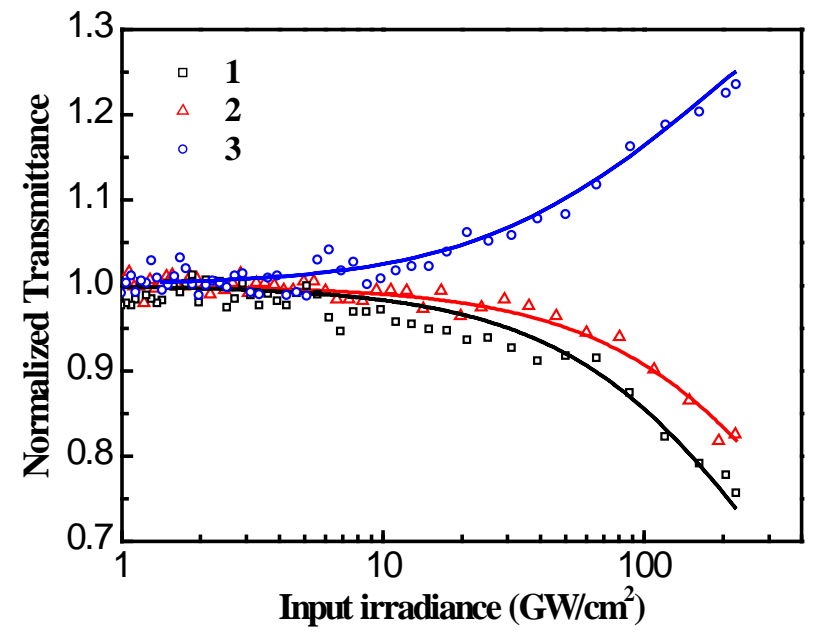

Fig. 6. Nonlinear transmission behaviors of the chloroform-methanol solutions of complexes with the concentration of $5.0 \times 10^{-4} \mathrm{M}$ as a function of the input irradiance under the femtosecond laser excitation at $800 \mathrm{~nm}$ wavelength. The scatters are the experimental results, while the solid lines are the theoretical simulations with the parameters obtained from Z-scan experiments. 